\title{
One-Dimensional Boson Fields in the Critical Range of EuS and EuO
}

\author{
U. KÖBLER* \\ Research Centre Jülich, Institute PGI, 52425 Jülich, Germany \\ (Received May 13, 2015; in final form July 14, 2015)
}

\begin{abstract}
Following the principles of renormalization group theory the typical experimental indications are discussed that in ordered magnets with a three-dimensional spin the dynamics of the spins is controlled by a boson guiding field instead by exchange interactions to the neighboring spins. The spins are, so to say, sensors to probe the dynamics of the relevant boson field. It is evident that these findings are not complementary but fundamentally different from atomistic concepts. The bosons are essentially magnetic dipole radiation emitted upon precession of the ordered moments. Within the individual domain the one-dimensional field has the character of a laser field. The field aligns all spins along its axis. In order that in cubic magnets three-dimensional dynamic symmetry can result a vector average over all one-dimensional boson fields of the individual domains is necessary. It is argued that this averaging process does not work in the critical temperature range of cubic EuS and EuO. As a result, the critical behavior of $\mathrm{EuS}$ and $\mathrm{EuO}$ is that of the one-dimensional boson field of the isolated domain and agrees with the critical behavior of the one-dimensional antiferromagnet $\mathrm{MnF}_{2}$. For the magnets with 1D boson field and half-integer spin it is found that the rational exponents $\beta=1 / 3, \gamma=4 / 3$ and $\nu=2 / 3$ give an excellent account of the mean exponent values over the most accurately known experimental data. These exponents obey the scaling relation $2 \beta=3 \nu-\gamma$.
\end{abstract}

DOI: $10.12693 /$ APhysPolA.128.398

PACS: 75.10.-b, 75.30.Ds

\section{Introduction}

One of the most remarkable results of renormalization group (RG) theory was to have convincingly proven that on approaching the magnetic ordering transition from the paramagnetic side spins and interactions between spins become unimportant for the dynamics (of the spins!) $[1,2]$. According to Brézin et al. the symmetry near the critical point is dynamically generated whatever interactions one takes [3]. In other words, the critical dynamics of the spins is not determined by exchange interactions to the neighboring spins. This conclusion has the severe consequence that atomistic models are inappropriate for the description of the critical spin dynamics. Even more serious is the conclusion that the actual value of the ordering temperature is also not determined by the exchange interactions and therefore is different from the ordering temperature expected by atomistic models. The magnetic ordering transition found by the algorithms of RG theory has been called stable fixed point (SFP) in order to distinguish it from the atomistic ordering transition. Much surprising is that RG theory finds a phase transition also when spins and interactions between spins are excluded from dynamics. This strange result poses the question: what drives the magnetic ordering transition (of the spins)? Independence of the SFP from the exchange interactions becomes obvious from the fact that in many magnets the observed transition temperature, the SFP, is at a much lower temperature than conforms

*e-mail: u.koebler@fz-juelich.de to magnon energies at zone boundary, the near neighbor interaction strengths. Equivalently, it is observed that magnons can persist much into the paramagnetic phase $[4,5]$.

When exchange interactions and spin degrees of freedom are of no importance on the dynamics (of the spins!) the dimensionalities of spins and exchange interactions also are of no importance. This necessitates a completely new classification scheme of ordered magnets. When interactions with neighboring spins are unimportant, the spins must receive their dynamics from somewhere else. The mysterious origin of the dynamically generated symmetry near the critical point [3] could, however, not be clarified by RG theory.

Complete unimportance of atomistic structures for the critical spin dynamics inevitably means that the dynamics is that of the continuous magnetic medium. In terms of RG theory, the new dynamic symmetry in the vicinity of the ordering temperature is invariance with respect of transformations of length scale. This is the definition of a continuum. Note that in a continuum there are neither atoms nor spins. This is well known from the elastic continuum that contains, by symmetry arguments, no atoms. Continuous translational invariance entails that the momenta of the excitations of the elastic continuum, the sound waves, are conserved quantities. In other words sound waves propagate to a good approximation ballistic, independent of the thermal motion of the atoms. Consequently the excitations of the continuum (sound waves) are independent and different from the excitations of the atomistic system (phonons). Just the analogous applies to the continuous and to the atomistic magnetic solid. Magnons are local excitations of the 
atomistic spin system, mainly due to nearest neighbor exchange interactions.

Quite generally, the excitations of a continuous medium are freely propagating bosons. We have called all types of bosons of the magnetic continuum GSW bosons giving tribute to Goldstone, Salam, and Weinberg [6]. In other words, the dynamics of the spins is controlled not by exchange interactions but by the GSW boson field. Conventional spin dynamics due to exchange interactions to the neighboring spins has to be replaced by the dynamics of the boson field. The spins are only weakly coupled to the field, and follow the dynamics of the field. Of course, interactions to the neighboring spins still exist but these interactions determine the non relevant magnon dispersions. As a consequence, atomistic spin models have to be replaced by a field theory. When all thermal energy is in the field, field theories need to consider the energy degrees of freedom of the field exclusively. However, before a field theory of magnetism can be formulated, precise knowledge of the field quanta is necessary. Unfortunately, RG theory could not specify the nature of the field quanta, the GSW bosons. Realistic field theories of magnetism therefore are not yet possible. Available field theories of the critical dynamics suffer from severe conceptual problems and are based on heuristic assumptions on the nature of the field [7]. The assumptions on the field have been made in such a way as to reproduce the critical exponents of the atomistic models that were considered as essentially correct. This is, of course, an inadequate motivation. A historically more serious consequence of this erroneous field concept was that RG theory appeared to be a method supplementary to the atomistic models.

Definite information on the nature of the field quanta is provided by the observation that only in genuine Ising magnets the spin dynamics is as predicted by atomistic models. A key experiment in this respect was a neutron scattering study of the sublattice magnetization of the 2D Ising antiferromagnet $\mathrm{Rb}_{2} \mathrm{CoF}_{4}$ [8]. Using neutron scattering it was shown that the sublattice magnetization of $\mathrm{Rb}_{2} \mathrm{CoF}_{4}$ precisely follows the Onsager exact solution of the atomistic $2 \mathrm{D}$ Ising model $[9,10]$. The $\mathrm{Rb}_{2} \mathrm{CoF}_{4}$ experiment allows one to conclude that the GSW field bosons are essentially magnetic dipole radiation emitted upon precession of the ordered magnetic moments. The field has radiation character! Since the Ising spins do not precess they are unable to generate field quanta (magnetic dipole radiation). The GSW boson field therefore gets not populated and the dynamics is, in fact, atomistic, that is, magnon defined.

Genuine Ising magnets are rare exceptions. For the large class of magnets with three-dimensional spin there are no exactly solved atomistic models available to compare the observed temperature dependence of the magnetic order parameter with theoretical predictions. Since closed analytical solutions as for the 2D Ising model are evidently not possible for systems with three-dimensional spins, atomistic models make power series expansions for

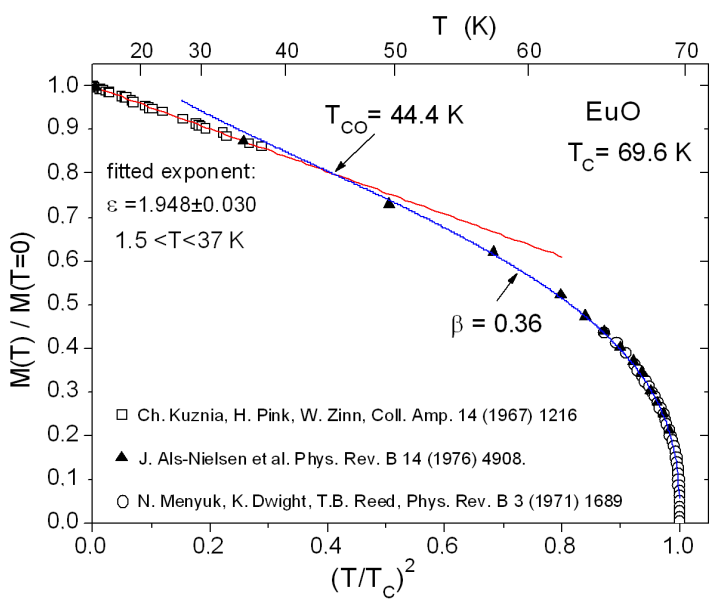

Fig. 1. Normalized zero field spontaneous magnetization data of $\mathrm{EuO}$ obtained by neutron scattering (triangles) [33] and by ${ }^{153} \mathrm{Eu}$ NMR (squares) [35] as a function of reduced temperature squared. Macroscopic spontaneous magnetization data (circles) are also depicted [36]. Low temperature $T^{2}$ universality class conforms to the expected isotropic dynamic symmetry. Critical exponent of $\beta \sim 1 / 3$ indicates one-dimensional boson field in the critical range.

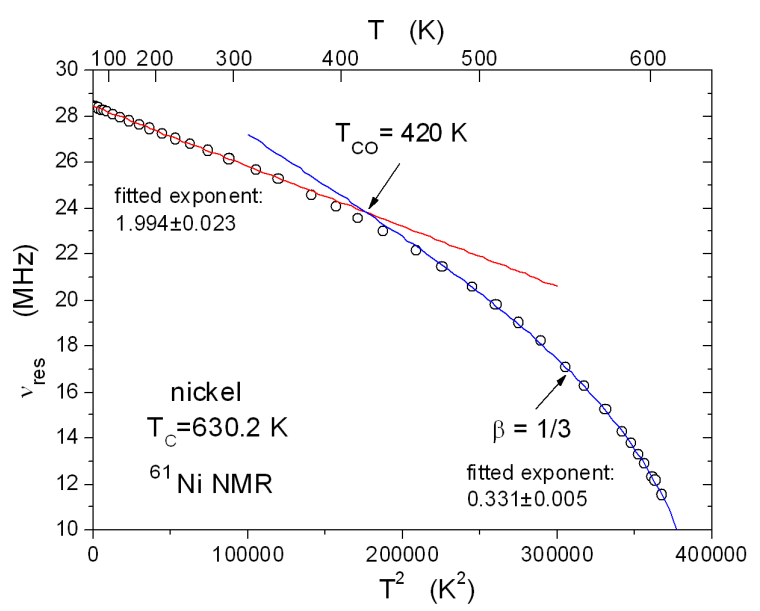

Fig. 2. Zero field ${ }^{61} \mathrm{Ni}$ NMR frequencies of bcc nickel as a function of absolute temperature squared [38]. Crossover from universal $T^{2}$ function to critical power function with exponent $\beta=1 / 3$ is at $T_{\mathrm{CO}}=420 \mathrm{~K}$. $T^{2}$ function means isotropic boson field $\left(S_{\text {eff }}=1 / 2\right)$ while $\beta=1 / 3$ is indicative of the $1 \mathrm{D}$ boson field. Critical range is as large as $\Delta T / T_{\mathrm{C}}=0.33$.

the thermal decrease of the order parameter at $T=0$ and for the rise of the order parameter at $T_{\mathrm{c}}$. Famous examples are the Bloch asymptotic $T^{3 / 2}$ function for the thermal decrease of the spontaneous magnetization of the isotropic ferromagnet and the Onsager critical power function $\sim\left(T_{\mathrm{N}}-T\right)^{1 / 8}$ for the atomistic $2 \mathrm{D}$ Ising model. The two power functions are the leading term of a power series expansion at $T=0$ and at $T=T_{\mathrm{N}}$, respectively. The width of the critical range of the atomistic models is, so to say, zero. This is in sharp contrast to the 
large validity range of the observed critical power functions due to field dynamics (see discussion of NMR data in Fig. 1 and Fig. 2). The observed critical power functions hold up to a considerable distance away from critical temperature. In fact, universality can be defined as the typical thermodynamic behavior of a field of freely propagating bosons. $T=0$ is also a critical temperature in the sense that the dynamics in the vicinity of $T \approx 0$ is due to a boson field. The "critical" power functions at $T=0$ are power functions of absolute temperature. It is now empirically well established that the two critical power functions at $T=0$ and at $T=T_{\mathrm{c}}$ overlap and give complete description of the magnetic order parameter for all temperatures in the ordered state $[11,12]$. In other words, the dynamics of ordered magnets (with a three-dimensional spin) never is controlled by atomistic magnons.

According to the different magnon dispersions of ferromagnets and of antiferromagnets atomistic spin wave theory expects that thermal decrease of the order parameter should be different for ferromagnets $\left(\sim T^{3 / 2}\right)$ and for antiferromagnets $\left(\sim T^{2}\right)$. This is in disagreement with the experimentally observed universality [11, 12]. Universality means a spin structure independent dynamics. In other words, the observed universal spin dynamics cannot be defined by magnons. Another strong experimental indication for field dynamics is that the dynamics is different in magnets with integer and half-integer spin [11-13]. This observation is also in contrast to atomistic spin wave theory. Since integer and half-integer spins precess differently they emit different types of field quanta. The dynamics of the field (its heat capacity) therefore is different for the two spin species.

Another very important term of RG theory is relevance. Relevance can be considered as a dynamic symmetry selection rule. When bosons are the relevant excitations all thermal energy is in the boson field. The spin system is thermodynamically not active and receives its dynamics through a weak coupling from the field. The coupling strength is given essentially by the emission probability for magnetic dipole radiation. Local interactions to neighboring spins (magnons) are atomistic details that are not relevant for the field dynamics and therefore can completely be ignored by field theories. Note that the field dynamics is independent of spin structure. It therefore suffices to consider the energy degrees of freedom of the field exclusively. This is an enormous analytical simplification (in principle). It is evident that the excitation spectrum of the field is different from the excitation spectrum of the atomistic magnons. Note that the only thermodynamic observable of a boson field is its heat capacity. Transfer of thermal energy from interacting spins to the boson field occurs at the crossover from the Curie-Weiss susceptibility to critical paramagnetic susceptibility. In the two temperature regions the susceptibility is described by different temperature functions. Typical for a crossover is an analytical change in the temperature dependence.
Since the coupling of the field bosons to the atomistic background is weak, the mean free path of the bosons is large. Note that the dispersion relation of freely propagating bosons is a simple power function of wave vector over a large range of energy. This results into a heat capacity of the field that is a simple power function of temperature over a large range of temperature. Thermal decrease of the magnetic order parameter with respect to saturation at $T=0$ is given by the heat capacity of the GSW boson field, and is identical for ferromagnets and for antiferromagnets of the same symmetry class. In other words, the temperature dependence of the heat capacity of the boson field can be measured in a twofold way, either by direct heat capacity measurements or by the thermal decrease of the magnetic order parameter. Note, however, that the heat capacity of the GSW boson field dominates the observed heat capacity only if it is larger than the heat capacity of the Debye boson field $\left(\sim T^{3}\right)$. This is the case if the ordering temperature is much lower than the Debye temperature [14]. A superposition of the heat capacity of the Debye boson field $\left(\sim T^{3}\right)$ and the heat capacity of the GSW boson field $\left(\sim T^{\varepsilon}\right)$ is not observed.

Generation of magnetic dipole radiation by magnetic moments is by stimulated emission along precession axis. The axial radiation characteristic provides vector character to the GSW boson field, and is an important source of magnetic anisotropy. As is well known, within any magnetic domain all spins are oriented parallel, quite independent of the anisotropy of the exchange interactions. We must assume that axial spin alignment is due to the coupling of the spins to the one-dimensional boson field. Within any domain the boson field resembles a laser field. Another example that the GSW boson field supports collinear spin order is provided by the amorphous ferromagnets. Amorphous ferromagnets with a sufficiently high ordering temperature exhibit collinear spin structure in spite of random local exchange anisotropies. If not too large, local anisotropies are not relevant and seem to be completely absent. Only for spin glasses local anisotropies are strong enough to be relevant.

Mono-domain 1D dynamic symmetry in bulk magnets is realized only in magnets with axial lattice structure such as $\mathrm{MnF}_{2}$ with tetragonal rutile structure. At the Néel transition of $\mathrm{MnF}_{2}$ only the longitudinal susceptibility diverges [15]. This characterizes the Néel transition as a one-dimensional phase transition. As a consequence of the one-dimensional boson field the $\mathrm{Mn}^{2+}$ moments are aligned parallel to tetragonal $c$-axis. Since magnon dispersions [16] as well as the spin of the $\mathrm{Mn}^{2+}$ ion are isotropic, it becomes evident that the ordering transition is not driven by magnons but by the onedimensional field. The surprisingly strong axial magnetic anisotropy that couples the magnetic moments rigidly to field axis (the crystallographic $c$-axis) is in sharp contrast to the isotropic spin and to the isotropic magnon dispersions of $\mathrm{MnF}_{2}$. The spin-flop field of $\mathrm{MnF}_{2}$ is as large as $120 \mathrm{kOe}$ [17]. As concerns the origin of the 
1D boson field we can only say that it must result from the axial (tetragonal) lattice structure. Note that the magnetic continuum has much similarity with the elastic continuum.

For cubic bulk magnets with an equal domain orientation along all three space directions, isotropic dynamic symmetry must be the result of an averaging process over all one-dimensional boson fields of the individual domains. This averaging process is completely unexplored by available field theories but it has the character of a vector sum. Condition for an average to be formed seems to be that the boson fields of neighboring domains are in dense contact with each other. The mean free path of the GSW bosons therefore must be at least as large as the linear dimension of the domains. In other words, the ratio between mean free path of the GSW bosons and linear dimension of the domains is an important mesoscopic parameter for field dynamics. This view could be proven as essentially correct by investigations of the ferromagnetic saturation process of a bcc iron sphere using neutron scattering [18]. Upon ferromagnetic saturation the domains with spin orientations parallel to field axis increase on the expense of the domains with a different spin orientation. Before the field for saturation is reached it happens that the size of the remaining domains becomes larger than the mean free path of the GSW bosons.

In Ref. [18] evidence of a field induced dimensionality crossover from isotropic to one-dimensional boson field has been obtained. Note that the saturated ferromagnet is in the mono-domain state and, in principle, 1D. In our context it is important to note that one-dimensional dynamic symmetry does not request that the whole sample is in the single domain state. It suffices that the domains are sufficiently large to be decoupled. $\mathrm{RbMnF}_{3}$ turned out to be a suitable example to illustrate that one-dimensional dynamic symmetry can be realized in a cubic bulk magnet. Magneto-elastic coupling is unusually weak in $\mathrm{RbMnF}_{3}$ [19]. As a consequence, magnetic domains are large. In fact, it is observed that thermal decrease of the sublattice magnetization of cubic $\mathrm{RbMnF}_{3}$ follows the same $T^{5 / 2}$ function as the sublattice magnetization of tetragonal $\mathrm{MnF}_{2}$ [20]. The $T^{5 / 2}$ universality class has been identified as characteristic for the magnets with $1 \mathrm{D}$ boson field and half-integer spin $[11,12]$. In other words bulk $\mathrm{RMnF}_{3}$ consists of a relatively small number of decoupled large domains and exhibits 1D dynamic symmetry of the isolated domain. It is evident that we have to assess the (dynamic) dimensionality of magnets to the dimensionality of the relevant GSW boson field and not to the dimensionality of the non relevant magnons. Curiously, cubic $\mathrm{RbMnF}_{3}$ has to be characterized as a one-dimensional bulk antiferromagnet in spite of isotropic magnon dispersions [21]. In other words, the dimensionality of the magnon dispersions can be different from the dimensionality of the boson field. In conformity with the spin structure independence of the GSW boson field, domain formation also is independent of spin structure. This illustrates that magnons are microscopic excitations on the length scale of the interatomic distance while GSW bosons are global excitations of the magnetic continuum. Since the two systems differ by translational symmetry (discrete and continuous) they have different dispersion relations and interact only weakly [22].

In $\mathrm{RbMnF}_{3}$ the mean free path of the GSW bosons seems to be shorter than the linear dimension of the domains for all temperatures. The observed GSW boson field is one-dimensional for all temperatures. It can, however, quite generally be assumed that the mean free path of the GSW bosons decreases as a function of increasing temperature. It therefore appears possible that the ratio between mean free path of the bosons and linear dimension of the domains falls below some critical value such that a dimensionality crossover from isotropic to one-dimensional boson field can occur as a function of increasing temperature. As experiments show, when this crossover occurs it does not manifests as a separate crossover event but coincides with the common crossover (at $T_{\mathrm{CO}}$ ) from universal $T^{\varepsilon}$ function at low temperatures to critical power function at $T_{\mathrm{c}}$. This crossover then assumes a twofold meaning. In other words it appears possible that in cubic magnets the universality class is isotropic in the range of the $T^{\varepsilon}$ function but onedimensional in the critical temperature range. The aim of this communication is to provide experimental evidence for this scenario using EuS and $\mathrm{EuO}$ as prominent examples.

On discussing critical exponents it is important to note that the observed critical exponents are due to field dynamics and, therefore, should not be compared with the atomistic model predictions. Most confusing is that the observed exponents at critical point often are quantitatively not much different from atomistic model predictions but they can occur in a strange context. For instance, the critical exponents of $\mathrm{MnF}_{2}$ with isotropic pure spin moment are close to the atomistic 3D Ising model predictions [15]. A much clearer distinction between atomistic exponents and field exponents is possible at $T=0$. For many cubic ferromagnets with half-integer spin (EuS, EuO, GdZn, $\mathrm{CrB}_{3}$ ) thermal decrease of the order parameter with respect to saturation at $T=0$ is by $T^{2}$ function and not by the Bloch atomistic $T^{3 / 2}$ function (see discussion of Fig. 1 and Fig. 2). As we have mentioned the universal $T^{\varepsilon}$ functions are independent of spin structure but they depend on the dimension $(d)$ of the field and on whether the spin quantum number is integer or half-integer. For $d=3,2,1$ the exponents are $\varepsilon=2,3 / 2$ and $5 / 2$ for magnets with half-integer spin but $\varepsilon=9 / 2,2$ and 3 for magnets with integer spin [22].

As experiments show, the critical exponent $\beta$ of the one-dimensional boson field is rather close to $\beta=1 / 3$, independent of spin quantum number. On the other hand, for the magnets with isotropic boson field the critical exponent is $\beta=1 / 3$ for integer spin but $\beta=1 / 2$ for half-integer spin. Quite generally, the mean field exponent seems to be characteristic for isotropic dynamic 
symmetry [23]. The difference to the atomistic mean field model is, however, that the critical power function of the boson dynamics holds up to a considerable distance away from $T_{\mathrm{c}}$

\section{Analysis of experimental data}

We start discussion of available experimental data for the magnetic order parameter by focusing on magnets with half-integer spin and isotropic boson field ( $T^{2}$ universality class). Isotropic boson field can occur also in non cubic magnets if (field) anisotropy is sufficiently weak and not relevant. Complete unimportance of an assumed finite but weak anisotropy proves stability of the universality classes. This is condition that all magnets can fit a few universality classes.

One of the historically first experimental identifications of the two universal power functions of $T^{2}$ and $\sim\left(T_{\mathrm{N}}-\right.$ $T)^{1 / 2}$ was reported for the weak ferromagnet $\mathrm{MnCO}_{3}$ with rhombohedral lattice structure [24]. The spin structure of $\mathrm{MnCO}_{3}$ is slightly canted antiferromagnetic such that in the basal plane a weak ferromagnetic component results. The ferromagnetic component with a saturation magnetic moment of only $0.033 \mu_{\mathrm{B}} / \mathrm{Mn}^{2+}$ allows one to study this antiferromagnet using macroscopic magnetization measurements. As early as in 1959 it was found that at low temperatures the spontaneous ferromagnetic moment follows $T^{2}$ temperature dependence and that the critical exponent is to a good approximation $\beta=1 / 2$. We can assume that the two exponents are representative also of the main antiferromagnetic component. As we will see, the two exponents $(\varepsilon=2, \beta=1 / 2)$ observed for $\mathrm{MnCO}_{3}$ are confirmed by other magnets with cubic lattice symmetry and half-integer spin and can be considered as standard behavior of this class of magnets. In particular, mean field critical exponent is typical for isotropic dynamic symmetry [23]. In other words, in $\mathrm{MnCO}_{3}$ no dynamic dimensionality crossover occurs in the range $0<T<T_{\mathrm{N}}$.

We should note that $\varepsilon=2$ and $\beta=1 / 2$ indicate an equivalent behavior at $T=0$ and at $T=T_{\mathrm{c}}$. Note that the $T^{\varepsilon}$ function describes the deviations from saturation at $T=0$. Equivalently, the deviations from $T_{\mathrm{c}}$ must be written as $T_{\mathrm{c}}-T \sim M^{1 / \beta}$. In other words $1 / \beta=\varepsilon$ means a formal identical behavior at $T=0$ and at $T=T_{\mathrm{c}}$.

Another example of the combination $\varepsilon=2$ and $\beta=$ $1 / 2$ is hexagonal $\mathrm{RbMnCl}_{3}$ with $S=5 / 2$ due to the $\mathrm{Mn}^{2+}$ ion [25]. Figure 3 shows normalized sublattice magnetization data obtained using neutron scattering as a function of reduced temperature squared. Scatter of the experimental data points is relatively high but, nevertheless, $T^{2}$ function at low temperatures and critical power function with mean field exponent $\beta=1 / 2$ are reasonably resolved.

A famous cubic magnet to further confirm the combination $T^{2}$ and $\sim\left(T_{\mathrm{N}}-T\right)^{1 / 2}$ is ferrimagnetic yttrium iron garnet (YIG) with sum formula $\mathrm{Y}_{3} \mathrm{Fe}_{5} \mathrm{O}_{12}[26,27]$. In YIG the spin of the $\mathrm{Fe}^{3+}$ ion is $S=5 / 2$. As for the weak ferromagnet $\mathrm{MnCO}_{3}$ the spontaneous

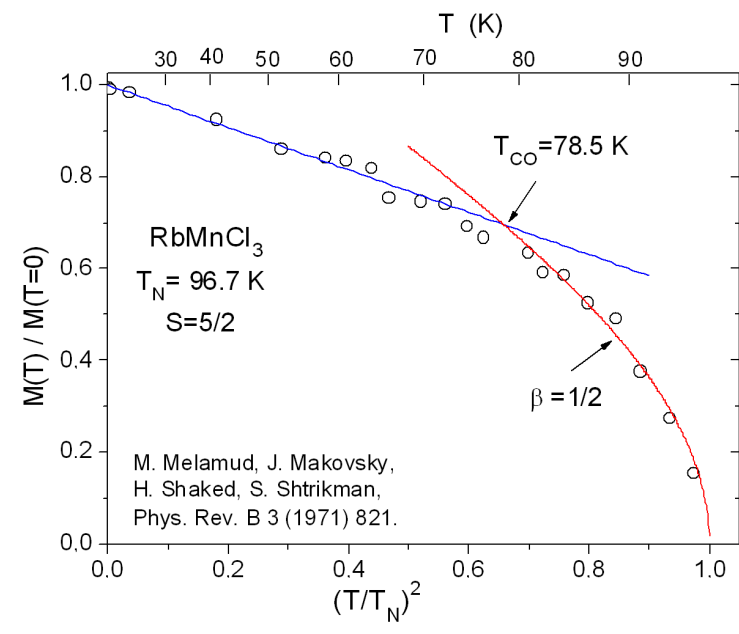

Fig. 3. Normalized order parameter of antiferromagnetic $\mathrm{RbMnCl}_{3}$ as a function of reduced temperature squared [25]. The typical critical power functions at $T=0, T^{2}$, and the critical power function of mean field type at $T_{\mathrm{N}}$ of the magnets with isotropic boson field and half-integer $\operatorname{spin}(S=5 / 2)$ are reasonably confirmed.

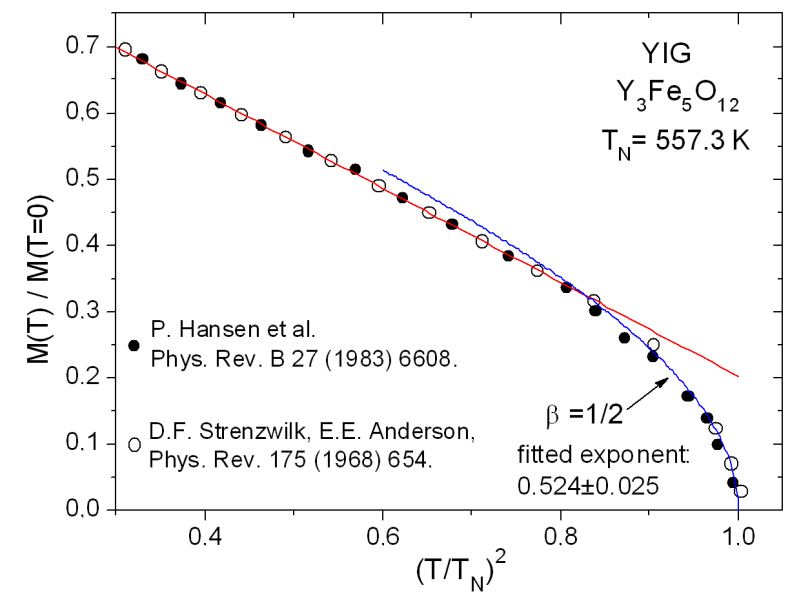

Fig. 4. Normalized spontaneous magnetization of the ferromagnetic component of ferrimagnetic $\mathrm{Y}_{3} \mathrm{Fe}_{5} \mathrm{O}_{12}$ (YIG) as a function of reduced temperature squared [26, 27]. The combination $T^{2}$ and $\sim\left(T_{\mathrm{N}}-T\right)^{1 / 2}$ is confirmed for this cubic ferrimagnet with $S=5 / 2$.

magnetization of YIG can be sampled on account of the ferromagnetic component using macroscopic magnetization measurements [28]. Figure 4 shows normalized spontaneous magnetization data of the ferromagnetic component as a function of reduced temperature squared. Crossover from low temperature $T^{2}$ function to critical power function of type $\left(T_{\mathrm{N}}-T\right)^{1 / 2}$ is apparent. We should note that for lower temperatures then are shown in Fig. 4 crossover to a second $T^{2}$ function with larger pre-factor follows [28]. We have called this type of crossover between identical power functions of absolute temperature amplitude crossover. In other words, the dynamic symmetry of YIG remains isotropic down to the 
lowest temperatures. In the spontaneous magnetization of YIG this amplitude crossover is at $T_{\mathrm{AC}} \approx 260 \mathrm{~K}[28]$. An amplitude crossover indicates that a non relevant parameter changes appreciably with temperature without getting relevant. It is a typical characteristic of RG theory that non relevant parameters enter the pre-factor of the universal power function only and are rather hidden. In other words, the universality classes are surprisingly stable. An amplitude crossover is a threshold induced event indicating a considerable (monotonous) change of a non-relevant parameter. Only when the non-relevant parameter changes strongly and finally becomes relevant a crossover to another universality class (exponent) is induced.

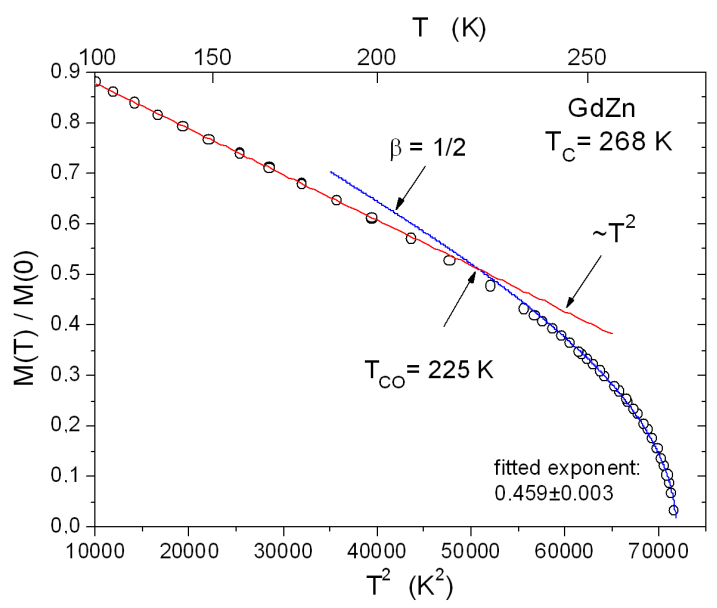

Fig. 5. Normalized macroscopic spontaneous magnetization of a spherical sample of the cubic ferromagnet GdZn [13] reflecting the typical behavior of a magnet with isotropic boson field and half-integer spin: the universal power function at $T=0$ is $T^{2}$ and the exponent of the critical power function is $\beta=1 / 2$. Isotropy is, however, not self evident in the state with ferromagnetic saturation (see text)

$\mathrm{GdZn}$ is a metallic ferromagnet with cubic lattice symmetry and spin $S=7 / 2$ due to the $\mathrm{Gd}^{3+}$ ion [29]. For not quite clear reasons, in macroscopic magnetization measurements on a spherical GdZn sample the isotropic exponents $(\varepsilon=2, \beta=1 / 2)$ are observed. Figure 5 shows normalized spontaneous magnetization data measured on a GdZn sphere as a function of absolute temperature squared. These data confirm $T^{2}$ universality class at low temperatures and critical power function of mean field type. Note the "normal" width of the critical range of $\Delta T / T_{\mathrm{C}}=0.16$.

Isotropy in macroscopic magnetization measurements is surprising. As we have mentioned, in the state of ferromagnetic saturation with all moments aligned parallel to field axis, one-dimensional behavior can be anticipated. We must assume that in GdZn in spite of ferromagnetic saturation the boson field is isotropic. This might be due to the spherical shape of the sample and due to a large mean free path of the GSW bosons. Diffuse scattering of the bosons on the inner surface of the sample can homogenize the boson field. Additionally scattering of the GSW bosons with conduction electrons also can contribute to an isotropic boson field. We should mention that the same universality classes as for GdZn are observed also in magnetization measurements of ferromagnetic $\mathrm{GdMg}[11,12]$. On the other hand, for fcc nickel isotropy is observed in zero field ${ }^{61} \mathrm{Ni} \mathrm{NMR}$ measurements but, as expected, 1D dynamic symmetry in the macroscopic magnetization (compare Fig. 2 and Fig. 6). This shows that correct interpretation of the observed universal exponents can be a complicated task.

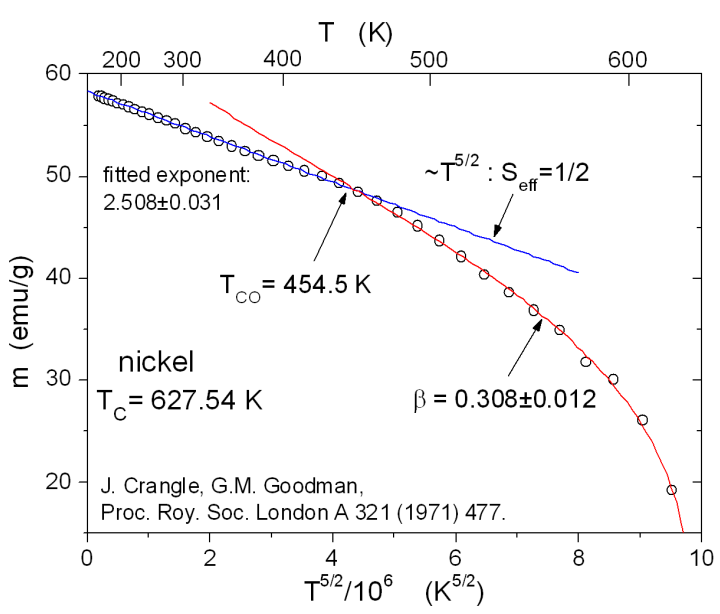

Fig. 6. Macroscopic spontaneous magnetic moment of nickel as a function of $T^{5 / 2}$ [31]. In the state of magnetic saturation nickel exhibits, as $\mathrm{MnF}_{2}$ (see Fig. 5), the universal exponents of a magnet with $1 \mathrm{D}$ boson field and half-integer (effective) spin $(\varepsilon=5 / 2$ and $\beta=1 / 3)$.

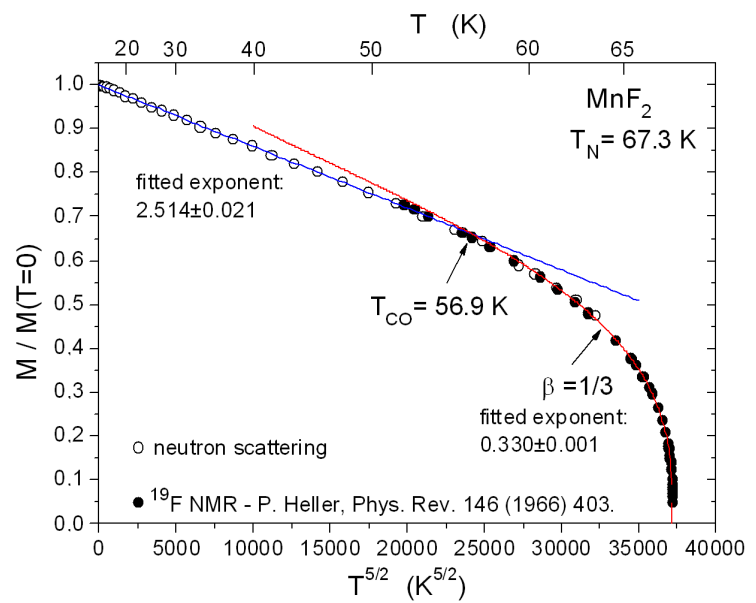

Fig. 7. Normalized order parameter of antiferromagnetic $\mathrm{MnF}_{2}$ reflecting the typical behavior of a magnet with one-dimensional boson field and half-integer spin: the universal power function at $T=0$ is $T^{5 / 2}$ and the critical power function has exponent $\beta=1 / 3[20,30]$.

We now discuss the typical behavior of magnets with one-dimensional boson field for all temperatures $\left(0<T<T_{\mathrm{N}}\right)$ and with half-integer spin, beginning with tetragonal $\mathrm{MnF}_{2}$ (Fig. 7) [15, 20]. The universality class 
at $T=0$ is $T^{5 / 2}$ and the critical exponent $\beta$ is to a very good approximation $\beta=1 / 3$ [30]. Figure 7 combines neutron scattering data (circles) [20] and ${ }^{19} \mathrm{~F}$ NMR data (dots) [30]. In order to avoid the rather severe extinction problems in single crystal experiments the neutron scattering data have been obtained on powder material. $\mathrm{MnF}_{2}$ unambiguously proves that the critical exponent of the one-dimensional boson field is $\beta=1 / 3$. As materials with integer spin show, $\beta=1 / 3$ applies to $1 \mathrm{D}$ magnets with integer spin as well [13].

A further example of the two universality classes $(\varepsilon=$ $5 / 2$ and $\beta=1 / 3)$ is nickel in the state of ferromagnetic saturation. As we have mentioned, in the state of ferromagnetic saturation 1D dynamic symmetry can be anticipated. By various reasons this expectation is not always confirmed. For the itinerant ferromagnet nickel with cubic bcc lattice structure 1D dynamic symmetry is confirmed in macroscopic magnetization measurements $[13,31]$. Comparison of Fig. 6 and Fig. 7 reveals identical exponents for ferromagnetically saturated nickel and for antiferromagnetic $\mathrm{MnF}_{2}$. Spin structure independence of the exponents again proves universality of the field dynamics.

As a conclusion, in the state of ferromagnetic saturation the itinerant metallic ferromagnet bcc nickel exhibits $1 \mathrm{D}$ dynamic symmetry as the insulating tetragonal antiferromagnet $\mathrm{MnF}_{2}$ with half-integer spin of $S=5 / 2$. It is not self-evident but experimentally well confirmed that the itinerant ferromagnets $\mathrm{Fe}, \mathrm{Ni}$, and $\mathrm{Co}$ can be classified by the same dynamic symmetry classes as magnets with localized magnetic moments. According to a saturation magnetic moment of $0.616 \mu_{\mathrm{B}} / \mathrm{Ni}$ it is reasonable to attribute an effective spin quantum number of $S_{\text {eff }}=1 / 2$ to nickel $[13,31]$. We should, however, note that the low temperature behavior of the macroscopic spontaneous magnetization of nickel (not shown in Fig. 6) is more complicated than reveals by Fig. $6[13,31]$. For reasons of completeness we should note that at about $120 \mathrm{~K}$ crossover to low temperature $T^{3 / 2}$ function can be identified in the data of [31]. $T^{3 / 2}$ universality class is consistent with $S_{\text {eff }}=1 / 2$ but belongs to the $3 \mathrm{D}$ anisotropic boson field. Crossover to a higher symmetry class at lower temperatures can, at least qualitatively, be explained by an increasing mean free path of the GSW bosons with decreasing temperature. The bosons then can average over larger regions of the sample. This, in principle, can result into a higher dynamic symmetry class at low temperatures.

As was demonstrated by Figs. 3-5, 8 (and by many more not discussed examples) there is ample experimental evidence that in the isotropic magnets with halfinteger spin the critical behavior is of mean-field type. The cubic ferromagnets EuS and EuO (Fig. 1, Fig. 9), and other examples to follow, obviously do not conform to this systematic. On the one hand, the anticipated low temperature $T^{2}$ function of the isotropic magnets with half-integer spin $(S=7 / 2)$ is confirmed for EuS and $\mathrm{EuO}$ but, on the other hand, the critical exponent $\beta$ is close to

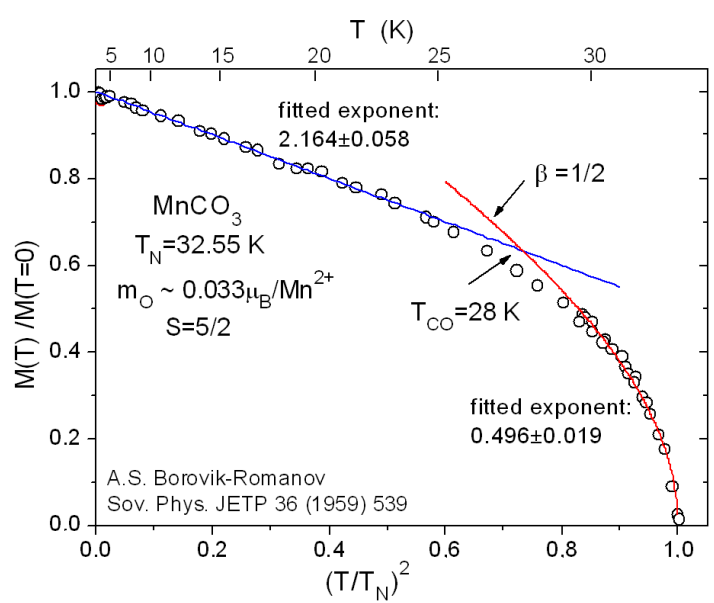

Fig. 8. Normalized ferromagnetic component of the weak ferromagnet $\mathrm{MnCO}_{3}$ as a function of reduced temperature squared [24]. Crossover from low temperature $T^{2}$ function to critical power function with mean field exponent of $\beta=1 / 2$ is at $T_{\mathrm{CO}}=28 \mathrm{~K}$. The width of the critical range, $\Delta T=T_{\mathrm{N}}-T_{\mathrm{CO}}$, is $\Delta T / T_{\mathrm{N}}=0.14$. The two exponents belong to isotropic dynamic symmetry.

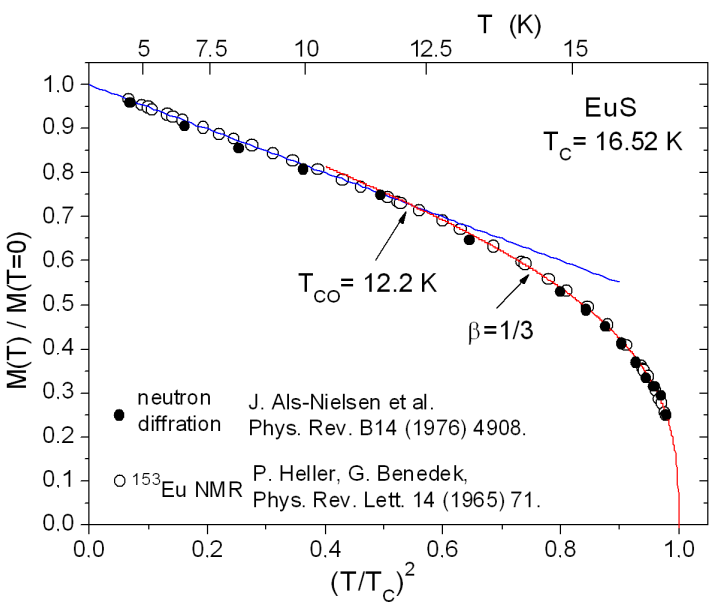

Fig. 9. Normalized zero field spontaneous magnetization data of EuS obtained by neutron scattering [33] and by ${ }^{153} \mathrm{Eu} \mathrm{NMR} \mathrm{[34]} \mathrm{as} \mathrm{a} \mathrm{function} \mathrm{of} \mathrm{reduced} \mathrm{tempera-}$ ture squared. Low temperature $T^{2}$ universality class conforms to the expected isotropic dynamic symmetry. Critical exponent of $\beta \sim 1 / 3$ is indicative of a onedimensional boson field. The dimensionality crossover from $3 \mathrm{D}$ to $1 \mathrm{D}$ boson field can be assumed to coincide with the crossover at $T_{\mathrm{CO}}=12.2 \mathrm{~K}$.

$\beta=1 / 3$ instead of $\beta=1 / 2$ [32]. In many investigations using zero field methods such as neutron scattering [33] and ${ }^{153} \mathrm{Eu} \mathrm{NMR} \mathrm{[34]} \mathrm{the} \mathrm{critical} \mathrm{exponent} \mathrm{of} \beta \approx 1 / 3$ has been confirmed for EuS and EuO. Since the exponent $\beta=1 / 3$ is well established for genuine $1 \mathrm{D}$ magnets it can safely be concluded that in EuS and EuO the boson field is $1 \mathrm{D}$ in the critical range but $3 \mathrm{D}$ below the critical range. An indirect indication that the assumed dimensionality crossover coincides with the common crossover from $T^{2}$ function to critical power function $\left(T_{\mathrm{C}}-T\right)^{1 / 3}$ 
is the enormous large critical range $\Delta T=T_{\mathrm{C}}-T_{\mathrm{CO}}$. For EuS $\Delta T / T_{\mathrm{C}}=0.26$ and for $\mathrm{EuO} \Delta T / T_{\mathrm{C}}=0.36$. A normal value for the critical range is $\Delta T / T_{\mathrm{c}}=0.15$. $1 \mathrm{D}$ dynamic symmetry in the critical range can be explained by a mean free path of the GSW bosons that has become shorter than the linear dimension of the domains. The domains then are decoupled and the bulk material exhibits 1D dynamic symmetry of the isolated domain. This situation applies to all temperatures in cubic $\mathrm{RbMnF}_{3}$ [20].

In Fig. 1 macroscopic magnetization data of $\mathrm{EuO}$ (circles) [36] have been depicted in addition to the zero field neutron scattering data (triangles) [33] and zero field ${ }^{153} \mathrm{Eu} \mathrm{NMR}$ data (squares) [35]. It can be seen that in the critical range zero field neutron scattering data and macroscopic magnetization data agree excellently. This is no surprise since the boson field is $1 \mathrm{D}$ in the zero field state and remains $1 \mathrm{D}$ in the state of magnetic saturation.

Nuclear magnetic resonance (NMR) is by far the most accurate method for the evaluation of the temperature dependence of the order parameter. Fit of $T^{\varepsilon}$ function over all available NMR data in the temperature range $0.0216 T_{\mathrm{C}}<T<0.536 T_{\mathrm{C}}[35]$ results in an exponent of $\varepsilon=1.948 \pm 0.030$ for EuO. In this temperature range the order parameter varies between $0.999<\nu / \nu_{0}<0.862$. The mean deviations from $T^{2}$ fit are 0.001. As a conclusion, the Bloch atomistic (non-universal) $T^{3 / 2}$ function is not identified using the most accurate method of NMR. Instead, data are satisfactorily described by universal $T^{2}$ function.

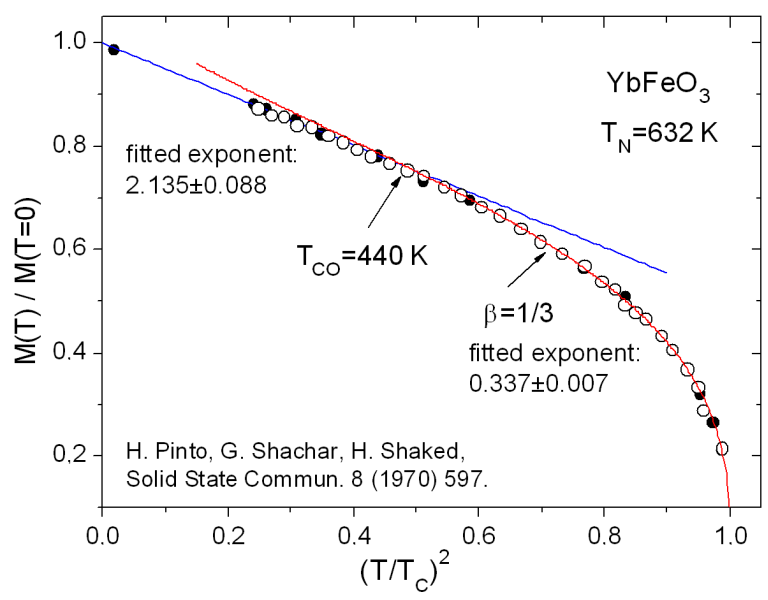

Fig. 10. Normalized order parameter of $\mathrm{YbFeO}_{3}$ measured by ${ }^{57} \mathrm{Fe}$ Mössbauer spectroscopy (dots) and by the method of neutron scattering (circles) as a function of reduced temperature squared [37]. Crossover from low temperature $T^{2}$ function to critical power function with $\beta=1 / 3$ is at $T_{\mathrm{CO}}=440 \mathrm{~K}$. The width of the critical range, $\Delta T=T_{\mathrm{N}}-T_{\mathrm{CO}}$, is $\Delta T / T_{\mathrm{c}}=0.30$.

In order to demonstrate that the unusual combination of the two universal power functions $T^{2}$ and $\left(T_{\mathrm{c}}-T\right)^{1 / 3}$ is not restricted to EuS and $\mathrm{EuO}$ we add $\mathrm{YbFeO}_{3}(S=5 / 2)$ and bcc nickel $\left(S_{\text {eff }}=1 / 2\right)$ as further candidates to illustrate the dimensionality crossover from 1D dynamic symmetry in the critical range to 3D dynamic symmetry below the critical range (Fig. 2, Fig. 10) [37, 38]. Exactly the same universal power functions as for $\mathrm{YbFeO}_{3}$, $\mathrm{EuS}$ and $\mathrm{EuO}$ are observed in zero field ${ }^{61} \mathrm{Ni}$ NMR measurements (Fig. 2). Fit of the exponent $\varepsilon$ to the ${ }^{61} \mathrm{NMR}$ frequencies in the temperature range $0.0075 T_{\mathrm{C}}<T<$ $0.55 T_{\mathrm{C}}$ results into exponent of $\varepsilon=1.994 \pm 0.023$. In this temperature range the normalized NMR frequencies (order parameter) vary between $0.999<\nu / \nu_{0}<0.889$. The mean deviations from $T^{2}$ fit function are 0.0005 .

Note the different behavior in the spontaneous magnetization of nickel (Fig. 6). In other words, upon magnetic saturation of nickel a dimensionality crossover from $T^{2}$ class in the zero field ground state to $T^{5 / 2}$ class in the state of ferromagnetic saturation occurs. The critical behavior is identical under the two experimental conditions.

\section{Conclusions}

We now are pretty sure that the critical exponent $\beta$ of the one-dimensional boson field is $\beta \approx 1 / 3$, independent of spin quantum number. Additionally, $\beta \approx 1 / 3$ is observed also for the isotropic magnets with integer spin. Only for the isotropic magnets with half-integer spin $\beta=1 / 2$. The frequent occurrence of the exponent of $\beta=1 / 3$ has already been noticed in 1966 [39]. In order to obtain a more accurate experimental average for the critical exponents of the magnets with half-integer spin and 1D boson field we now know that it is allowed to include data of the cubic magnets $\mathrm{RbMnF}_{3}, \mathrm{EuS}$ and $\mathrm{EuO}$ in addition to $\mathrm{MnF}_{2}$. This is important since the critical exponents of these materials are among the best known ones.

It is obvious that in $\mathrm{MnF}_{2}$ the boson field is onedimensional. Since spin and exchange interactions [16] are three-dimensional and isotropic in $\mathrm{MnF}_{2}$ the dimensionality of the boson field must somehow come from the tetragonal lattice symmetry. In other words, spin dimensionality and exchange dimensionality are unimportant for the dimensionality of the field. As a consequence, classification of magnets according to spin and exchange dimensionality is inappropriate. Table compiles experimental data for the critical exponents $\beta$ (order parameter), $\gamma$ (susceptibility) and $\nu$ (inverse correlation length) for $\mathrm{EuO}[32,33,36], \operatorname{EuS}[32,33,34], \mathrm{MnF}_{2}$ [15], and for $\mathrm{RbMnF}_{3}$ [40]. Within the typical error of \pm 0.02 the exponents of all four listed compounds agree very well.

Because the exponents $\varepsilon$ of the low temperature $T^{\varepsilon}$ universality classes are rational numbers there is some motivation to expect that the critical exponents also are rational numbers. This idea is supported by the exponents of Table. In fact, the exponent values averaged over all four materials are close to the rational numbers $\beta=1 / 3, \gamma=4 / 3$ and $\nu=2 / 3$. These idealized rational numbers obey the scaling relation $2 \beta=3 \nu-\gamma$. This is, however, a minimal condition only. Using the scaling relation $\alpha+2 \beta+\gamma=2$ a critical exponent of 
TABLE

Mean experimental values of critical exponents of magnets with one-dimensional boson field and halfinteger spin. Errors for all exponents are \pm 0.02 .

\begin{tabular}{c|c|c|c}
\hline \hline & $\beta$ & $\gamma$ & $\nu$ \\
\hline $\mathrm{EuO}$ & 0.36 & 1.32 & 0.68 \\
$\mathrm{EuS}$ & 0.34 & 1.28 & 0.70 \\
$\mathrm{MnF}_{2}$ & 0.35 & 1.27 & 0.634 \\
$\mathrm{RbMnF}_{3}$ & 0.32 & 1.366 & 0.70 \\
Idealized & $1 / 3$ & $4 / 3$ & $2 / 3$
\end{tabular}

$\alpha=0$ results from the exponents of Table. This value is in fair agreement with the averaged experimental value of $\alpha \approx 0.05 \pm 0.01$ [32]. We must, however, consider that the heat capacity is an integral quantity. Possibly, non magnetic contributions modify the value of the critical exponent $\alpha$. Furthermore, using the exponents of Table the scaling relation $\gamma=\beta(\delta-1)$ results into $\delta=5$. This value is definitively larger than the mean experimental value for $\mathrm{EuO}$ and $\mathrm{EuS}$ of $\delta=4.34 \pm 0.1$ [32]. As a conclusion scaling relations including the exponent $\delta$ of the critical isotherm are violated. In other words, the non intrinsic, field induced "critical" behavior does not conform to conventional scaling relations. This appears plausible since an external magnetic field acts not only on the spin but can act additionally on the field bosons if the bosons have magnetic moment. This is likely to be the case according to the non-linear $\sim q^{1.5}$ dispersion relation of the one-dimensional boson field [22]. Using $\alpha=0$ the same value of $\delta=5$ results from scaling relation $\alpha+\beta(\delta+1)=2$.

Comparison of the here proposed rational exponent values with available field theoretical calculations [7] appears inappropriate because these calculations are based on inadequate field concepts. At the time of these calculations the nature of the field and its action on the spin system were not yet sufficiently explored to formulate a realistic field theory of the critical dynamics. In particular no distinction is made between magnets with integer and half-integer spin quantum number. As experiments show, $\beta=1 / 2$ for the magnets with isotropic boson field and half-integer spin but $\beta=1 / 3$ for the isotropic magnets with integer spin. Development of reliable field theories of magnetism remains a big challenge for the future. The main conceptual drawback of the present field theories is that they retain the atomistic classification of the critical behavior in terms of dimensionality of the spin and dimensionality of the exchange interactions. In other words unimportance of the atomic degrees of freedom for the field dynamics was not respected consequently. As the example of $\mathrm{MnF}_{2}$ clearly shows classification of the critical dynamics must be exclusively according to the symmetry of the GSW boson field. In $\mathrm{MnF}_{2}$ the boson field is one-dimensional but spin and magnon dispersions are isotropic. It is due to the $1 \mathrm{D}$ boson field that only the $z$-component of the $\mathrm{Mn}^{2+}$ spin orders [15]. In other words, the field executes the phase transition. Ordering of only the $z$-component of the $\mathrm{Mn}^{2+}$ spin does not mean that $\mathrm{MnF}_{2}$ is an Ising system. The perfect paramagnetic isotropy [41], and the observed universality clearly disprove Ising behavior. The ordered spin component in $\mathrm{MnF}_{2}$ is pinned to the vector of the boson field. This situation applies to all domains. Within any domain the boson field is that of a laser.

Most surprising is that the one-dimensional boson field of the individual domain can define the dimensionality of cubic bulk magnets [20]. When the boson fields of the differently oriented domains are not in dense contact with each other they do not interact, and the bulk sample exhibits the dynamic behavior of the isolated domains and is one-dimensional. In one-dimensional $\mathrm{MnF}_{2}$ the whole bulk sample is one single domain. As a conclusion, the axial fields of the domains are the components of the global field. Isotropy of the global field results by some vector coupling of the one-dimensional field components.

Since the field is the relevant excitation spectrum future field theories of magnetism need to consider the energy degrees of freedom of the field exclusively. Thermal decrease of the order parameter is given by the heat capacity of the field. Curiously, the spin system - although the origin of magnetism - is not responsible for its own dynamics. This most remarkable conclusion of RG theory provokes a philosophical parable. Because the spin system has transferred all its thermal energy to the field (by giving field quanta into the field) it has lost its autonomy. This reminds on processes in the monetary marked. When everybody gives his money to the bank, the bank controls all money and the individual savers have no longer influence on what their money is doing but have to accept what the monetary marked prescribes.

\section{References}

[1] K.G. Wilson, J. Kogut, Phys. Rep. 12C, 75 (1974).

[2] K.G. Wilson, Rev. Mod. Phys. 47, 773 (1975).

[3] E. Brézin, J.C. Le Guillou, J. Zinn-Justin, Phys. Rev. B 10, 892 (1974).

[4] P. Martel, R.A. Cowley, R.W.H. Stevenson, Can. J. Phys. 46, 1355 (1968).

[5] J. Skalyo, Jr., G. Shirane, S.A. Friedberg, H. Kobayashi, Phys. Rev. B 2, 4632 (1970).

[6] J. Goldstone, A. Salam, S. Weinberg, Phys. Rev. 127, 965 (1962).

[7] J.C. Le Guillou, J. Zinn-Justin, Phys. Rev. B 21, 3976 (1980).

[8] H. Ikeda, K. Hirakawa, Solid State Commun. 14, 529 (1974).

[9] L. Onsager, Phys. Rev. 65, 117 (1944).

[10] C.N. Yang, Phys. Rev. 85, 808 (1952).

[11] U. Köbler, A. Hoser, Physica B 362, 295 (2005).

[12] U. Köbler, A. Hoser, Eur. Phys. J. B 60, 151 (2007).

[13] U. Köbler, A. Hoser, Renormalization Group Theory - Impact on Experimental Magnetism, Springer, Berlin 2010

[14] U. Köbler, A. Hoser, Acta Phys. Pol. A 127, 350 (2015). 
[15] M.P. Schulhof, R. Nathans, P. Heller, A. Linz, Phys. Rev. B 4, 2254 (1971).

[16] A. Okazaki, K.C. Turberfield, R.W.H. Stevenson, Phys. Lett. 8, 9 (1964).

[17] Y. Shapira, S. Foner, Phys. Rev. B 1, 3083 (1970).

[18] U. Köbler, A. Hoser, C. Thomas, J. Magn. Magn. Mater. 321, 1202 (2009).

[19] D.T. Teaney, V.L. Moruzzi, B.E. Argyle, J. Appl. Phys. 37, 1122 (1966).

[20] U. Köbler, A. Hoser, J. Magn. Magn. Mater. 325, 87 (2013).

[21] D.H. Saunderson, C.G. Windsor, G.A. Briggs, M.T. Evans, E.A. Hutchison, IAEA Conf. Proc. 1972, p. 639.

[22] U. Köbler, Acta Phys. Pol. A 127, 1694 (2015).

[23] U. Köbler, A. Hoser, J. Magn. Magn. Mater. 349, 88 (2014).

[24] A.S. Borovik-Romanov, Sov. Phys. JETP 36, 539 (1959).

[25] M. Melamud, J. Makovsky, H. Shaked, S. Shtrikman, Phys. Rev. B 3, 821 (1971).

[26] D.F. Strenzwilk, E.E. Anderson, Phys. Rev. 175, 654 (1968).

[27] P. Hansen, K. Witter, W. Tolksdorf, Phys. Rev. B 27, 6608 (1983).

[28] U. Köbler, A. Hoser, Solid State Commun. 142, 350 (2007).
[29] R. Aléonard, P. Morin, J. Pierre, D. Schmitt, Solid State Commun. 17, 599 (1975).

[30] P. Heller, Phys. Rev. 146, 403 (1966).

[31] J. Crangle, G.M. Goodman, Proc. R. Soc. Lond. A321, 477 (1971).

[32] U. Köbler, Ch. Sauer in: Landolt-Börnstein, Vol. III/12c, Eds. K.-H. Hellwege, A.M. Hellwege, Springer, Berlin 1982, p. 173.

[33] J. Als-Nielsen, O.W. Dietrich, L. Passell, Phys. Rev. B 14, 4908 (1976).

[34] P. Heller, G. Benedek, Phys. Rev. Lett. 14, 71 (1965).

[35] Ch. Kuznia, H. Pink, W. Zinn, Coll. Amp. 14, 1216 (1967).

[36] N. Menyuk, K. Dwight, T.B. Reed, Phys. Rev. B 3, 1689 (1971).

[37] H. Pinto, G. Shacher, H. Shaked, Solid State Commun. 8, 597 (1970).

[38] J. Englich, Charles University, Prague, private communication.

[39] M. Eibschütz, S. Shtrikman, D. Treves, Solid State Commun. 4, 141 (1966).

[40] A. Tucciarone, H.Y. Lau, L.M. Corliss, A. Delapalme, J.M. Hastings, Phys. Rev. B 4, 3206 (1971).

[41] J.W. Stout, L.M. Matarrese, Rev. Mod. Phys. 25, 338 (1953). 